\title{
Peripheral Nerve Grafting in the Spinal Cord: A Histological and Electrophysiological Study
}

J. Wardrope, M.B.Ch.B., F.R.C.S. and D. H. Wilson M.B.Ch.B., F.R.C.S. Research Registrar, Consultant in Accident and Emergency, General Infirmary at Leeds, Great George St, Leeds LS1 3EX, U.K.

\section{Summary}

Excision of the dorsal columns was used to create a lesion in the spinal cord. The defect was bridged using a peripheral nerve graft. Silver staining showed the grafts being invaded by axons. Neuronal tracing by injecting horseradish peroxidase into the graft showed the origin of these fibres to be in dorsal root ganglia and in the grey matter of the lumbar cord. All of the animals studied showed horseradish peroxidase staining of neurones in the grey matter of the lumbar cord caudal to the graft. Five of the animals had staining of the dorsal root ganglion cells.

Electro-physiological assessment was performed 5 to 6 months following the grafting procedure. Evoked potentials were recorded from the graft in six animals and from the dorsal columns above the graft in six animals.

Key words: Axonal regeneration; Cat; Horseradish peroxidase; Peripheral nerve grafts; Electrophysiological monitoring.

\section{Introduction}

Cajal used free peripheral nerve grafts to study the reactions of the central nervous system (CNS). He found that these grafts readily survived in the spinal cord and that neurones would grow into them (Cajal, 1928).

In 1940 Sugar and Gerard investigated the function in peripheral nerve grafts. They used rats as the model and used a variety of materials as the grafts. They found evidence of functional improvement in a small proportion of the animals with evidence of regenerating tracts in animals treated with peripheral nerve grafts. They also performed electrophysiology on these animals and found some evidence for conduction of action potentials (Sugar and Gerard, 1940).

The advent of microsurgical techniques allowed a far greater precision in nerve grafting and in the 1970s Kao began to examine the subject. He noted that there was still much glial scarring with cavitation at the cord graft junctions. He evoked Cajal's concept of 'spinal cord autonomy' as the inevitable response 
of the cord to trauma. He went on to study the time course of events in the cord and found that the lytic enzymes released by the damaged axons were no longer found in the extra cellular space a week following the injury. He also claimed that the terminal clubs which produced these enzymes were stabilised within the week and thus he introduced the concept of delayed spinal cord grafting (Kao and Chang, 1977). Waiting for a week after the trauma to insert the graft appeared to prevent cyst formation (Kao et al., 1977, Wrathnal et al., 1982).

The 1980s saw a further step in the investigation of these grafts with application of neuronal tracing techniques to investigate the origin of axons which are found in these grafts. The major workers in this area have been Aguayo and his colleagues in Montreal. They have used horseradish peroxidase and autoradiography to study the axons in peripheral nerve grafts. They have shown that spinal grafts are populated with axons originating in the dorsal root ganglia but also from cells in the grey matter in the spinal cord (David and Aguayo, 1980) and in the brain stem (Richardson et al., 1984). However they also have provided very elegant evidence of the failure of cortico-spinal fibres to enter the grafts. They injected $\mathrm{C} 14$ labelled amino acids into the motor cortex of animals previously grafted and were able to demonstrate the pyramidal tracts up to the rostral cord graft junction. There was no radio-activity seen in the graft (Richardson et al., 1982).

Some workers have found that these grafts conduct electrical impulses (Sugar and Gerard, 1940) but others have refuted this (Brown, 1947; Barnard and Carpenter, 1950). Recent encouraging work from others and from our own laboratories (Wilson, 1984) has prompted us to investigate the function of these grafts. Previous work on spinal grafting has used the model of spinal cord transection. The validity of this model has been challenged since spinal cord transection is rare in clinical practice and transection may impose strains on the cord graft junctions which hinder neuronal regeneration (Breig, 1982). We therefore have used the model of dorsal column excision and grafting as a pilot study into the functioning of peripheral nerve grafts in the spinal cord.

\section{Methods}

Ten female cats weighing $2 \cdot 5-3 \mathrm{~kg}$ were used in the experiment. Anaesthesia was induced with intramuscular Althesin (alphaxalone/alphadolone acetate) and maintained by intravenous Althesin. A laminectomy was performed at the thoraco-lumbar junction to expose the L2 segment of the spinal cord. Using the operating microscope a one centimetre segment of the dorsal columns was excised. The removed sections of the cord were weighed and these varied from $0.23 \mathrm{~g}$ to $0.48 \mathrm{~g}$. A section of the sensory branch of the radial nerve was obtained from the forelimb and placed in the trough left by the excision of the dorsal columns. The dura, posterior vertebral muscles and skin were closed and the animals allowed to recover.

The animals were nursed on sheepskin with 8 hourly bladder care and passive exercises to the hind limbs.

Five to 6 months following the initial procedure the animals were reanaesthetised. Following induction by intramuscular Althesin a $3 \mathrm{~mm}$ endotracheal tube was inserted and the animal ventilated using oxygen and nitrous 
oxide. Anaesthesia was maintained using intravenous Althesin delivered by syringe pump and intravenous Haemaccel used for fluid replacement.

The operation site was re-opened and a laminectomy performed rostral and caudal to the graft site which was left undisturbed at this stage. The left sciatic nerve was exposed in the thigh and a stimulating electrode placed around it. The animal was placed in a spinal frame and a neuro-muscular blocking drug (pancuronium, $0.06 \mathrm{mg} / \mathrm{kg}$ ) given. The effect of the pancuronium was allowed to wear off before the next dose was given to ensure adequate anaesthesia was being maintained. The animal was monitored throughout the procedure.

Using a Neuralog pulse generator a signal of $1 \mathrm{~Hz}$ at $0.5-9.0 \mathrm{~mA}$ and 100 microseconds pulse width was applied to the sciatic nerve electrode. A platinum electrode (Neuralog) mounted on a NL I00 headstage was used to record from the dorsal columns. The response was amplified and displayed on a storage oscilloscope and recorded on a four-track Racal tape recorder.

An injection of 0.5 microlitres of a $20^{\circ}{ }_{0}$ solution of Horse Radish Peroxidase (HRP) was made into the graft and the animals were then sedated for 24-48 hours. They were then given a lethal dose of anaesthetic and perfused with glutaraldehyde/paraformaldehyde solution followed by cold $10^{\circ}{ }_{0}$ sucrose in phosphate buffer (Mesulam, 1982).

The lumbar cord, the lumbar dorsal root ganglia and the brainstem were removed. The lumbar cord was cut into $5 \mathrm{~mm}$ slices and the tissue for horseradish peroxidase staining was stored overnight in the sucrose buffer solution. The graft area for silver staining was placed in neutral formol saline. The tissue for HRP was frozen and cut at 40 micrometers on a cryostat. Sections were then processed using the tetra methyl benzidine method (Mesulam).
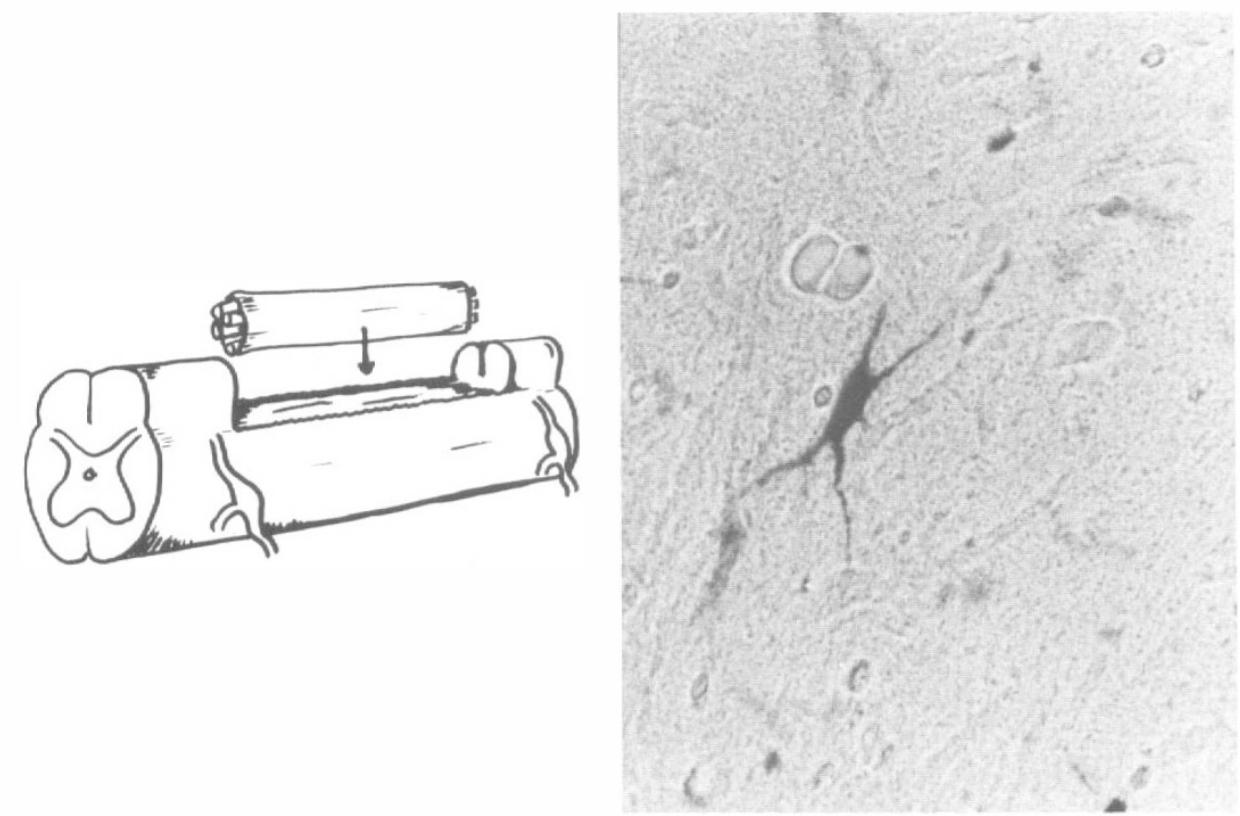

Figure 2. A neurone in the lumbar cord $5 \mathrm{~mm}$

Figure 1. Diagram of the grafting procedure. caudal to the cord graft junction well stained by the $\operatorname{HRP}(\times 250)$. 
The silver staining was carried out on paraffin sections cut at 15 micrometres using Holmes method (Drury). Serial sections were also stained using Weigerts' haematoxylin and Van Gieson's stain for collagen.

The sections of dorsal root ganglia, spinal cord and brainstem were searched for HRP positive neurones. Since artefact was a major problem in some sections of the spinal cord only cells with definite nuclei and processes were counted (Fig. 2). The maximum number of cells per section was noted for each of the 5 $\mathrm{mm}$ slices of cord and of each dorsal root ganglion.

\section{Results}

Following the initial grafting procedure most of the animals soon recovered bladder function and movement in the hind limbs. Two of the animals did not recover complete control of their bladders but careful attention to bladder expression and perineal toilet prevent any major excoriation of the skin.

\section{Silver staining and general histology}

Adequate silver stains were obtained on five animals. These showed the grafts to be well vascularised and sitting in the dorsal part of the cord (Fig. 3). Most of

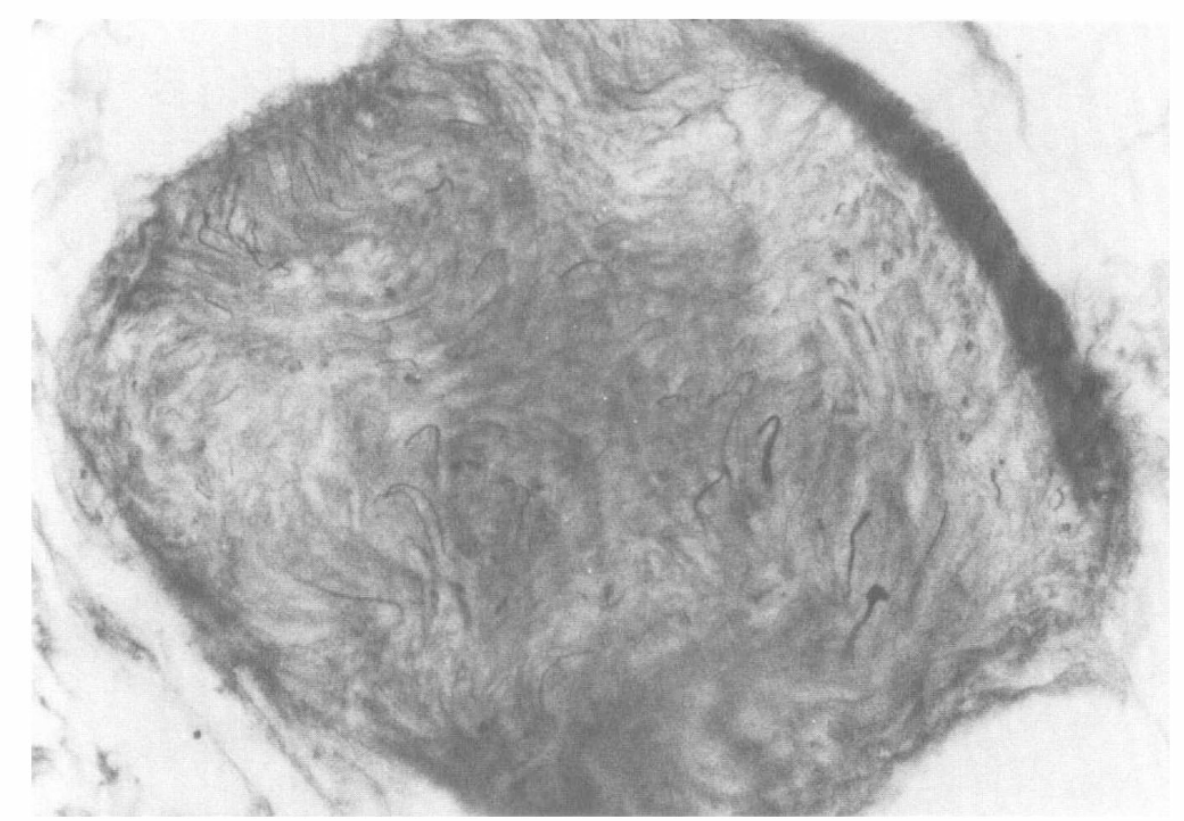

Figure 3. The dorsal region of the cord showing the graft fasicles (Silver stain $\times 50$ ).

the fasicles are well populated with neurones but fibre counts varied from no fibres seen per fasicle to forty fibres per fasicle cross section. The size of the fibres varied from 1 to 5 micrometres. Figure 4 shows a fasicle sectioned slightly obliquely showing the silver staining fibres. 


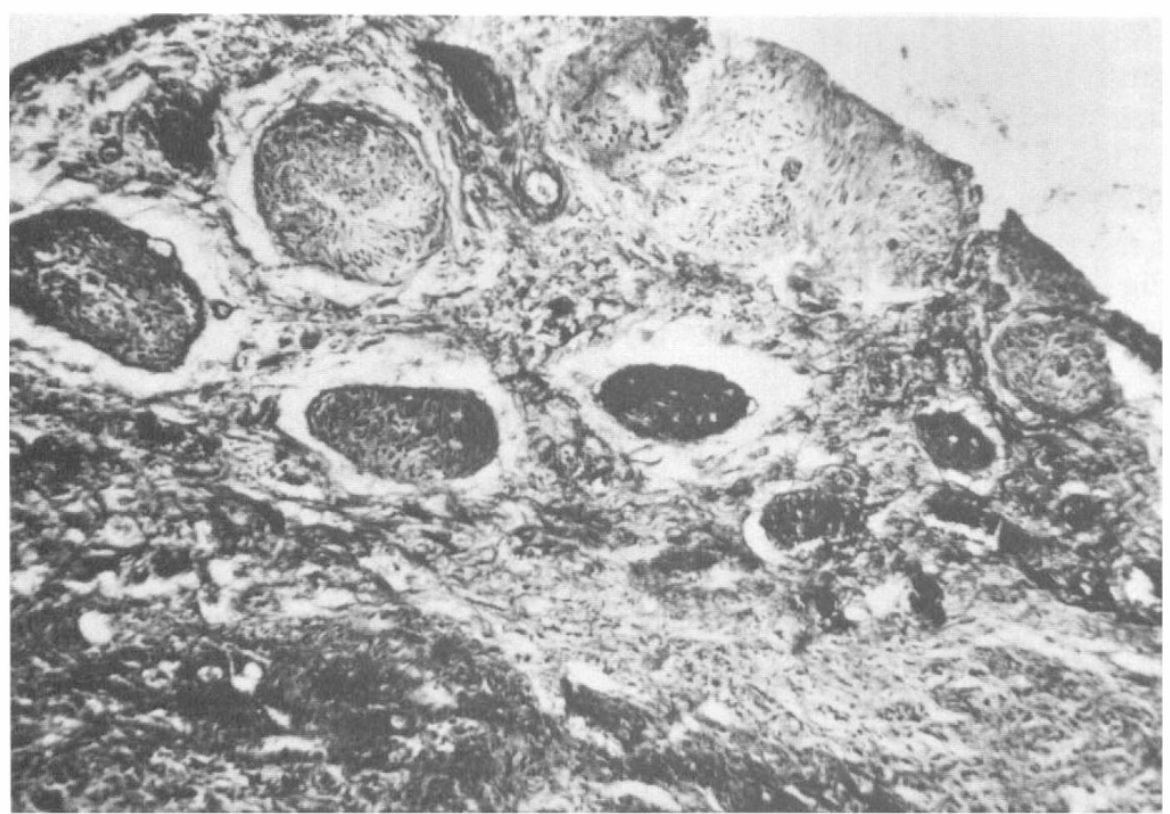

Figure 4. Graft fasicle longitudinal section showing plentiful fibres (Silver stain $\times 250$ ).

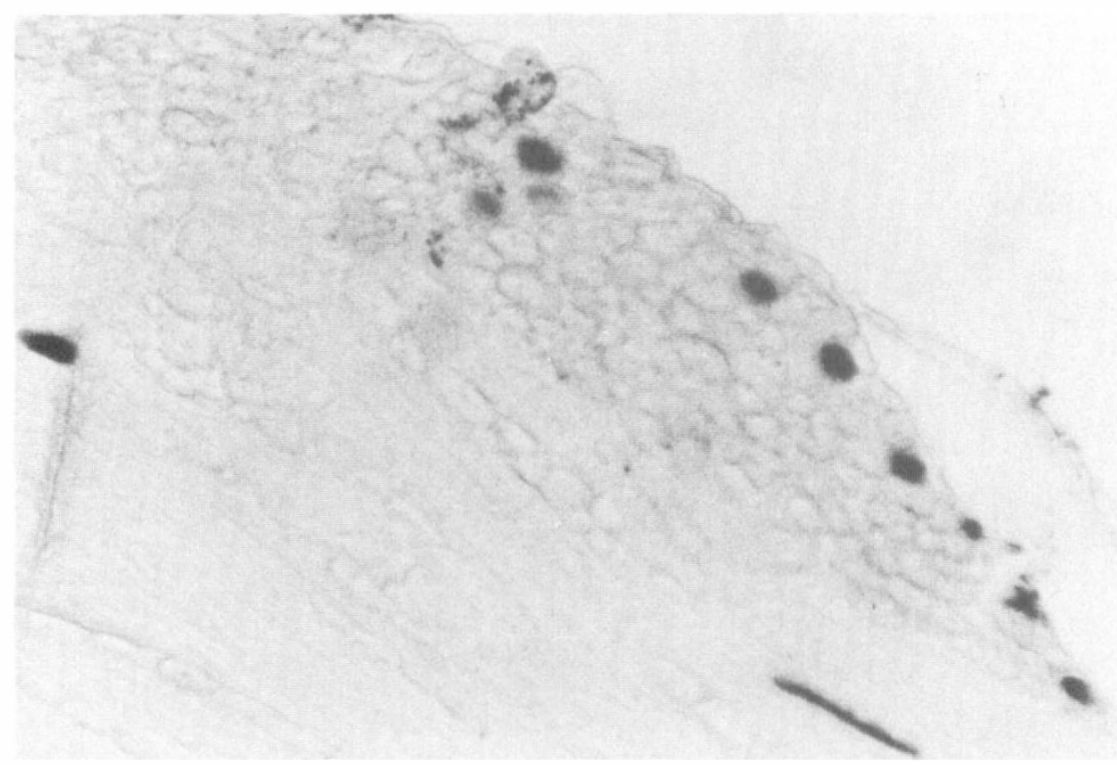

Figure 5. Dorsal root ganglion L6 showing ganglion.

Previous authors have found cyst formation to be a problem in this type of experiment (Kao, 1976) but we found a cyst at the cord graft junction in only one animal (cat 28). 


\section{Horseradish peroxidase study}

Satisfactory sections were obtained in seven animals. In two of these animals there was evidence of spread of the HRP outwith the graft (cats 29 and 38).

Dorsal root ganglia. HRP was found in the ganglion cells in five of the animals (Fig. 5). The two animals showing no staining had the shortest survival times following injection of the HRP. The ganglia showing the positive staining were one or two segments below the caudal cord graft junction. In one animal there was staining in a ganglion rostral to the graft.

Lumbar cord. All the animals had evidence of neuronal staining in the grey matter of the lumbar cord caudal to the graft. The numbers of neurones seen varied markedly from animal to animal (Table I). The numbers of neurones

Table I

\begin{tabular}{ccccc}
\hline & & \multicolumn{2}{c}{$\begin{array}{c}\text { Distance from Caudal } \\
\text { Cord Graft Junction }\end{array}$} \\
\cline { 3 - 5 } CAT & DRG & $0-5 \mathrm{~mm}$ & $5-10 \mathrm{~mm}$ \\
\hline 28 & 7 & 8 & 1 & \\
29 & 2 & 20 & 11 & (EG HRP) \\
33 & 4 & 4 & 0 & \\
35 & 1 & 10 & 0 & \\
36 & 0 & 10 & 3 & \\
37 & 4 & 7 & 4 & \\
38 & 0 & 10 & 5 & (EG HRP) \\
\hline
\end{tabular}

Maximum number of labelled neurones per section of dorsal root ganglion (DRG) and of two segments of spinal cord caudal the graft site. EG HRP indicates the animals in which free HRP was seen out with the graft.

decreased rapidly with distance from the cord graft junction. No labelled neurones were seen further than $10 \mathrm{~mm}$ from the cord graft junction. The neurones were mostly found in the dorsal horn and intermediate regions of the cord (Laminae IV, V and VI of Rexed).

Thoracic cord and brainstem. There were no labelled cells or evidence of axonal HRP in the brain stem. In one animal there was staining of three cells in the thoracic grey matter but this animal also had evidence of HRP spread outwith the graft (cat 29).

\section{Electrophysiology}

Electrophysiology was performed on eight animals (one animal died under anaesthesia and we were instructed to sacrifice one early as it lost part of its tail).

Figure 6 shows the type of recordings obtained from the three sites; one with the recording electrode in the dorsal columns of the lumbar cord caudal to the graft, secondly with the electrode in the graft and thirdly in the lower thoracic cord rostral to the graft.

Table II lists the results for each animal with the latency of the signal given in msecs. Recordings of evoked potentials were obtained in the dorsal columns above the graft in six of the eight animals. 

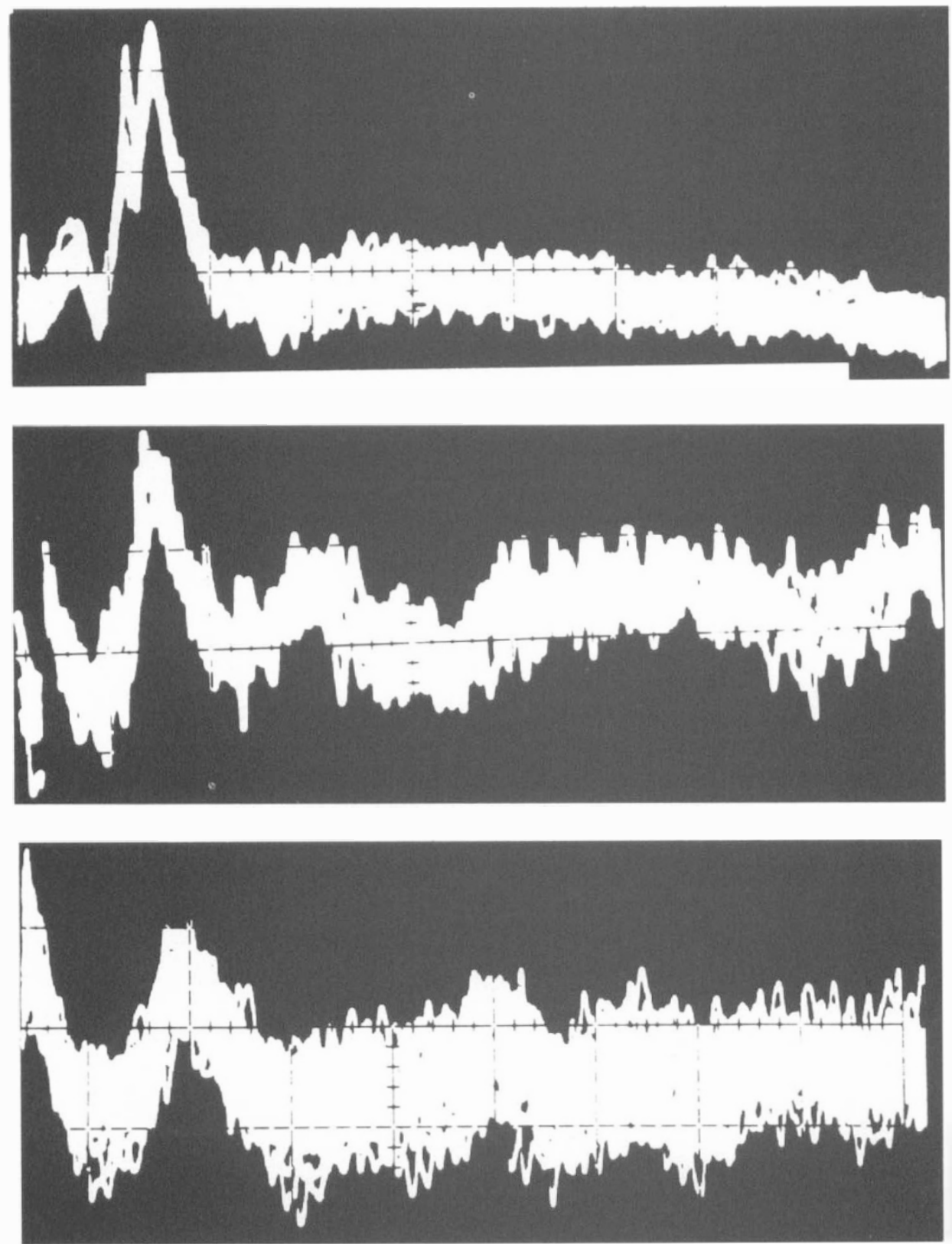

Figure 6. Records of the evoked potentials measured in response to sciatic nerve stimulation: a) Dorsal columns of the lumbar cord caudal to the graft. Latency $1.1 \mathrm{msec}$; b) Graft. Latency $1.2 \mathrm{msec}$; c) Dorsal columns of the cord rostral to the graft. Latency $1.5 \mathrm{msec}$.

\section{Conclusions}

The study confirms the work of others (Kao, 1977; Richardson, 1982) that peripheral nerve grafts survive in the spinal cord and encourage the growth of axons in damaged areas of the cord. 
Table II

\begin{tabular}{cccc}
\hline & \multicolumn{3}{c}{ Latency (msecs) } \\
\cline { 2 - 4 } CAT NO. & $\begin{array}{c}\text { Lumbar } \\
\text { Cord }\end{array}$ & Graft & $\begin{array}{c}\text { Thoracic } \\
\text { Cord }\end{array}$ \\
\hline 28 & 1.8 & 3.0 & N.R. \\
29 & 3.0 & N.R. & N.R. \\
33 & 2.4 & 2.8 & 3.5 \\
34 & 2.0 & 3.0 & 6.0 \\
35 & 3.0 & N.R. & 5.2 \\
36 & 2.0 & 2.2 & 2.4 \\
37 & 1.1 & 1.2 & 1.5 \\
38 & 1.5 & 1.7 & 2.0 \\
\hline
\end{tabular}

Results of evoked potential studies. Latency times of potentials recorded in the lumbar cord caudal to graft in the graft and in the cord rostral to the graft. (N.R. = not recorded).

The finding that many of the fibres are of dorsal root origin would be expected since the dorsal columns contain predominantly first order sensory neurones. However, the dorsal columns also carry some second order sensory neurones (Brown, 1981) and some descending fibres. This might explain the presence of HRP labelled neurones in the grey matter of the lumbar spinal cord. Thus it would appear that adult CNS neurones are indeed capable of sprouting axons in response to injury and that these axons will elongate into peripheral nerve grafts.

We did not find any evidence of HRP in the brainstem nucleii. This may have been due to technical factors such as the distance between the HRP injection site and the brainstem and the difficulties of picking up small amounts of HRP in dendrites.

We conclude that peripheral nerve grafts encourage the elongation of CNS fibres following section but we have found no evidence as of yet that these fibres make functional connections.

We have shown that it is possible to record evoked potentials from the graft and from the dorsal columns rostral to the graft in $75^{\circ}{ }_{0}$ of the animals studied.

However since a complete cord lesion has not been created the interpretation of the recorded potentials is difficult. It is possible that the potentials are originating in the cord anterior to the graft. The next stage of the experiment would be to create a complete cord lesion by crushing the cord as in the Allen technique and subsequently to graft the damaged area.

We conclude that the results of this pilot study reveals some of the difficulties in interpretation of the results of grafting the spinal cord without having created a complete lesion. There is no doubt that the grafts survive within the cord but it will be necessary to create a complete paraplegia to determine conclusively whether or not the graft provides a functional bridge between undamaged areas above and below the lesion.

\section{Acknowledgements}

We would like to thank the International Spinal Research Trust who funded the project and support for JW. 
We would also like to thank the Department of Anatomy, University of Leeds Medical School for the use of histological and computing facilities, $\mathrm{Mr} \mathrm{A}$. Trimble for his advice on histological technique and Dr D. G. F. Harriman for his help in the interpretation of the histological material. Dr J. Morrison, Dept. of Physiology for his advice regarding the electrophysiological measurements and $\mathrm{Mr}$ Hopkins and his colleagues in Laboratory Animal Services of Leeds Medical School.

\section{References}

BARNARD JW, CARPENTER W 1950 Lack of regeneration of the spinal cord of the rat. Journal of Neurophysiology 13:223-228.

Breig A, Renard M, Stefanko S, et al. 1982 Healing of the severed spinal cord by biomechanical relaxation and surgical immobilisation. Anatomia Clinica 4:167-181.

BRown AG, FyfFe REW 1981 Post synaptic dorsal column neurones. Journal of Physiology (Lond) 321:31-47.

BRown JO, McGough P 1947 Abortive regeneration of the transected spinal cord. Journal of Comparative Neurology 87:131-137.

DruRy RAB, Wallington EA 1976 The nervous system. In Carlton's Histological Technique, ed 4. Oxford University Press, London 260-262.

KaO CC, ChANG LW, BloodworTh JMB 1976 Axonal regeneration across transected mammalian spinal cord. An electron microscopic study of delayed nerve grafting. Experimental Neurology 54:591-615.

KaO CC, Chang LW, Bloodworth JMB 1977 Axonal regeneration across transected mammalian spinal cord. An electron microscopic study of delayed nerve grafting. Experimental Neurology 54:591-615.

Mesulam MM 1982 Principles of horseradish peroxidase neurohistochemistry and their applications for tracing neural pathways-axonal transport, enzyme histochemistry and light microscopic analysis. In: Mesulam MM Tracing neural connections with horseradish peroxidase. Wiley Interscience, Chichester, 1-157.

RAMON Y CAJAL 1928 Degeneration and regeneration in the nervous system. p589 Oxford University Press, London.

Richardson PM, MCGuinness UM, Agauyo AJ 1982. Peripheral nerve autografts to the rat spinal cord: studies with axonal tracing methods. Brain Research 7:147-162.

Richardson PM, Issa MK, Aguayo AY 1984 Regeneration of long spinal axons of the rat. Journal of Neurocytology 13:165-182.

SUgAR O'GeRARD RW 1940 Spinal cord regeneration in the rat. Journal of Neurophysiology 3:119.

WILSON DH 1984 Peripheral nerve implants in the spinal cord in experimental animals. Paraplegia 22:230-237. 